\title{
Acciones y herramientas de software para implementar entornos de inclusión educativa en Colombia
}

\author{
Actions and software tools to implement educational inclusion \\ environments in Colombia
}

Recibido: 15 Ago. 2017 - Revisado: 30 Sep. 2017

Aceptado: 30 Oct 2017 - Publicado: 30 Dic 2017

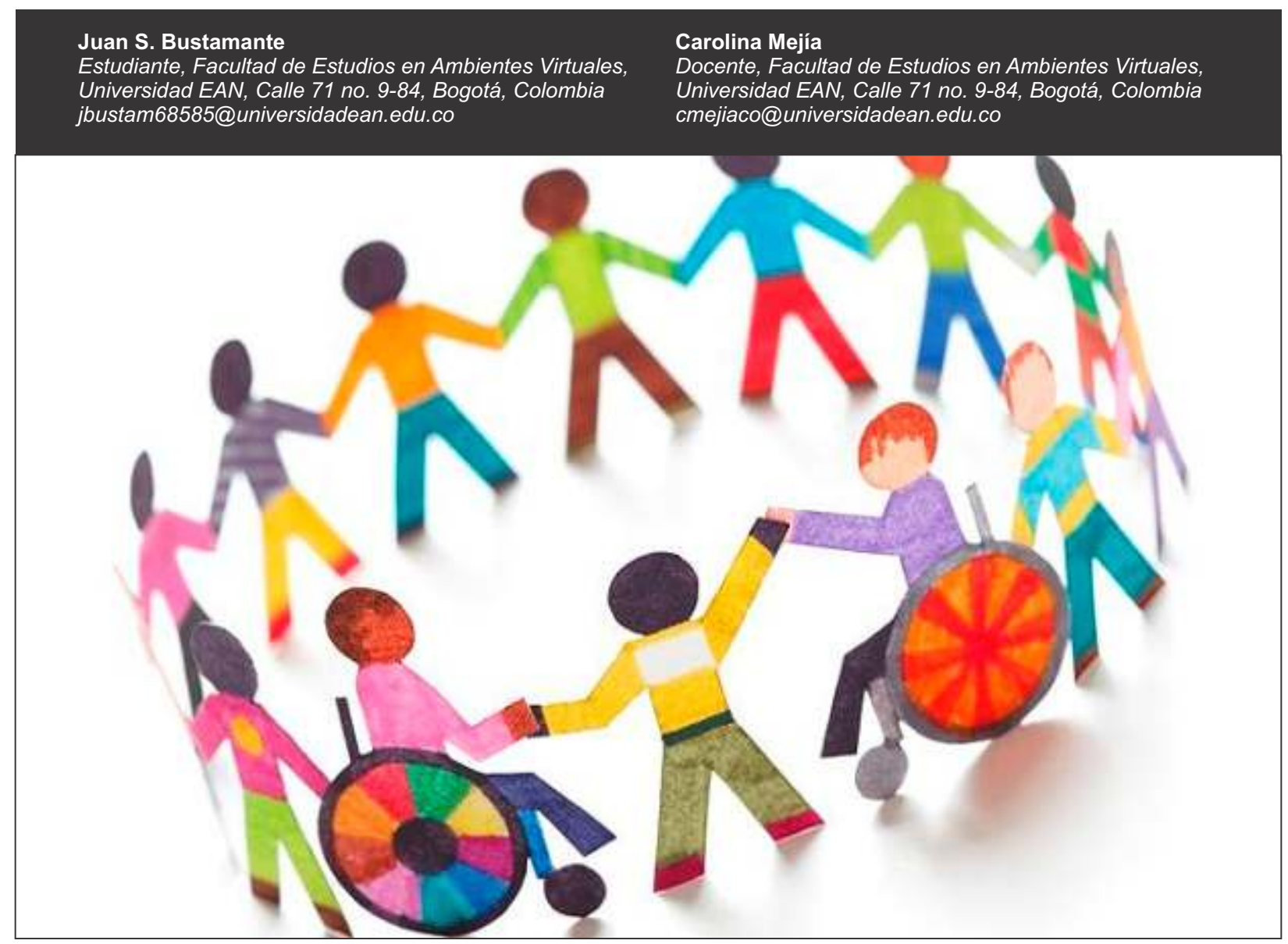

Resumen Una educación inclusiva promueve el acceso equitativo y la permanencia de los estudiantes con el fin de garantizar igualdad de oportunidades para todos. Las implementaciones pueden estar basadas tanto en estudios como en prácticas empíricas, y se enfocan principalmente desde tres aspectos: lo físico, lo cognitivo y lo social. Es así, como se hace relevante identificar y analizar casos exitosos y mejores prácticas realizadas por las instituciones educativas colombianas. De igual manera, dados los avances tecnológicos, es imperativo lograr una fusión entre la tecnología y la inclusión con el fin de llegar al objetivo deseado.

Palabras claves educación, inclusión, tecnología, UDL

Abstract: An inclusive education promotes equitable access and permanence of students in order to guarantee equal opportunities for all. The implementations can be based on both studies and empirical practices, and are mainly focused on three aspects: the physical, the cognitive and the social. This is how it is relevant to identify and analyze successful cases and best practices carried out by Colombian educational institutions. Similarly, given technological advances, it is imperative to achieve a fusion between technology and inclusion in order to achieve the desired objective.

Key words education, inclusion, technology, UDL 


\section{INTRODUCCIÓN}

La educación ha tenido diversas transformaciones a lo largo de la historia del ser humano, siempre tendientes a la búsqueda o generación de nuevos conocimientos que han sido transmitidos a través de las diferentes sociedades y generaciones. Sin embargo, la masificación del conocimiento sólo se logra durante el último siglo, dando la posibilidad de que más personas accedan a los saberes que se han logrado desarrollar.

En Colombia, es el Estado, en cabeza del Ministerio de Educación Nacional -MEN-, el directamente responsable de suscitar espacios y acciones que promuevan el acceso equitativo y de permanencia en el sistema educativo. Las Tecnologías de la Información y Comunicación -TICson herramientas poderosas para facilitar y mejorar la calidad de vida de las personas que por sus condiciones físicas se topan con barreras para acceder de manera ecuánime al sistema educativo; sin embargo, su existencia o uso per sé no es suficiente. Para que ellas realmente se conviertan en herramientas generadoras de igualdad de oportunidades educativas y de pertinencia en contenidos se requiere de políticas públicas que potencialicen su adaptabilidad a las personas con Necesidades Educativas Especiales -NEE- que así lo demandan.

El objetivo de este trabajo es socavar estudios e investigaciones que agrupen programas de educación inclusiva con el fin de identificar y analizar casos de éxito; así como las mejores prácticas utilizadas en las instituciones educativas colombianas. Adicional a ello, profundizar en aquellos recursos educativos que posibilitan y simplifican el acceso equitativo a las personas que encuentran impedimentos de acceso a la educación igualitaria, generando de esta manera espacios inclusivos en el proceso de aprendizaje.

De esta manera, el artículo se desarrollará indicando algunas generalidades de la educación inclusiva, seguido de casos de aplicación en Colombia y las estrategias utilizadas, para finalmente visualizar herramientas de software que apoyan los espacios de inclusión.

\section{GENERALIDADES DE LA EDUCACIÓN INCLUSIVA}

El punto de partida es la Declaración Universal de Derechos Humanos de 1948, donde se estipula el derecho que todas las personas tienen a la educación. Para la UNESCO, "las escuelas deben acoger a todos los niños, independientemente de sus condiciones físicas, intelectuales, sociales, emocionales, lingüísticas u otras" (1994), convirtiendo así al sistema educativo en un sistema inclusivo, basándose en el principio de que la pedagogía debe centrarse y adaptarse a las necesidades del estudiante y no que el estudiante deba adaptarse a las condiciones del lugar de enseñanza. Por tal razón, se crea una integración social en ambos sentidos, de la sociedad hacia las personas con NEE y de éstas hacia la sociedad.

El Estado colombiano, a través del MEN (2009), busca "el fortalecimiento a la capacidad institucional para la atención a la diversidad con el fin de desarrollar alternativas que permitan educar con calidad y equidad al creciente número de estudiantes que presentan habilidades personales o condiciones culturales diferentes al promedio de la población y que han sido objeto de exclusión del servicio educativo y por ende de una participación activa de la vida económica, social, política y cultural de sus comunidades". El primer paso consiste en generar una categorización del público objetivo de la educación inclusiva.

Así mismo, el Ministerio de Tecnologías de la Información y las Comunicaciones de Colombia MinTIC- desarrolla una serie de estrategias cuyo objetivo principal es el uso adecuado de la tecnología para el mejoramiento de la calidad de vida de todos los colombianos. Entre ellas se pueden contar 1) las capacitaciones en el uso de TIC para el teletrabajo, impartidas a personas privadas de la libertad en cárceles como Distrital y El Buen Pastor, en Bogotá, y Establecimiento Penitenciario de Mediana Seguridad y Carcelario de Tunja; 2) la alianza con el Instituto Nacional para Ciegos - INClpara la ejecución del proyecto 'Covertic', cuyo objetivo es que, a través de los programas Magic y Jaws, personas con limitaciones visuales puedan acceder a contenidos digitales e Internet desde sus computadores y 3 ) la identificación, a través de una insignia ubicada en el home de aquellos portales Web del país que sean inclusivos, para que los visitantes puedan saber si el sitio electrónico que están consultando es accesible a la población en situación de discapacidad, como se puede constatar en la página de Discapacidad Colombia (s.f).

\section{CASOS APLICADOS DE INCLUSIÓN}

\subsection{Básica Primaria y Secundaria}

A continuación, una síntesis de los casos aplicados de inclusión en Colombia, algunos de ellos, reseñados por Claudia Camacho (2013) en una nota especial para el periódico El Espectador. 
(a) Santa Mariana de Jesús es un colegio incluyente de Bogotá, donde su rectora, la hermana Yolanda López, recuerda el primer caso exitoso de inclusión en su plantel educativo, el de "una niña sin cerebelo tocó las puertas del colegio". Así como lo expresa la rectora, al principio no se sabía mucho del tema, sin embargo, decidieron afrontar el reto y para ello desarrollaron el siguiente plan: "primero capacitar tanto a profesores como a personal administrativo y de servicios". Se convirtió en una reinversión del profesor, exigiendo innovación, creatividad, cambios de paradigmas y otros en sus modelos pedagógicos. Segundo, la implementación del modelo 'dulce acompañante', que consiste en que los estudiantes con necesidades especiales asisten a clases regulares con una especie de 'lazarillo', quien favorece el proceso cognitivo.

Las conclusiones del modelo de educación inclusiva pueden verse en las siguientes ideas, expresadas por la hermana Yolanda: "en el colegio hay inclusión porque nosotros nos vemos desde la individualidad, y nos vemos como el niño que viene de la India, nos vemos como el niño autista, nos vemos como los niños afroamericanos, nos vemos como los niños que son más claritos y nos vemos en nuestra diferencia, eso es colombianidad, eso es ser marianita, es para lo que nosotros significa inclusión. Y a nivel social pedagógico, de la mano de los niños discapacitados hemos aprendido tolerancia, respeto por la diferencia".

(b) República Bolivariana de Venezuela es una institución educativa de Bogotá, que inició sus procesos de educación inclusiva hace alrededor de 20 años, en la escuela primaria Samper Mendoza, que posteriormente se fusionó con el colegio República Bolivariana de Venezuela, cuando un grupo de profesoras bajo la batuta de Margarita Posada, decidieron trabajar con niños autistas, convirtiéndose en uno de los pocos colegios públicos de Bogotá en incluir en el aula regular a niños con esta condición. Su objetivo es formar estudiantes autónomos e independientes, que puedan desarrollar sus propios proyectos de vida y para ello, en la institución, se incluyen a todos los estudiantes en el aula regular, mediante una flexibilización curricular llevada a cabo por los maestros y los docentes de apoyo, quienes a su vez apoyan a los niños en el manejo de las cartillas y las guías que han diseñado especialmente para ellos. (Camacho 2013).

Siguiendo con la autora, la educación inclusiva "hace que los niños de desarrollo típico sean mejores personas: son niños y jóvenes con gran sensibilidad, una valoración por la vida y una altísima educación ciudadana". Estos niños y jóvenes, por ejemplo, enseñan día a día que el diálogo es la mejor forma para solucionar los problemas. A nivel institucional, "se trata de entender que todos los niños tienen derecho a ser como cualquiera y a ser tratados como tal, con sus derechos y deberes".

(c) Desde el año 2003 el colegio Nuevo Gimnasio de Bogotá ha puesto en marcha el modelo de educación inclusiva. Empezó de modo intuitivo, de autoformación, algo de ensayo y error. Posteriormente, asistieron a un seminario de formación en Gordon Porter, Brunswick, Canadá. A nivel educativo, cuando una niña ingresa, se hace un trabajo de sensibilización con el equipo de apoyo, los maestros, los padres de las alumnas y las alumnas con las que va a compartir su vida escolar. Las niñas asisten al aula regular, en donde los maestros, con la ayuda del grupo de profesores, diseñan estrategias de grupo de trabajo para cada una de ellas. La finalidad del equipo de apoyo es enseñar a los profesores, no a las estudiantes. Generar sinergias a nivel interior de tal manera que todas las estudiantes compartan una vida académica de acuerdo a sus capacidades, sin hacer currículos diferenciadores ni excluyentes, en el mismo salón, con los mismos maestros y el mismo conocimiento, sin bajar el nivel para los niños regulares (Camacho 2013).

El reto de la educación inclusiva, como lo expresa Luz Stella Uricoechea, rectora del Nuevo Gimnasio, es "vencer los miedos, porque esos miedos no nos dejan avanzar. Es el miedo a la calificación del examen de Estado, el miedo a los estándares de los primeros puestos, el miedo a que se piense que la institución baja su nivel académico".

(d) El proyecto de educación inclusiva en la Institución Educativa Los Portales, en Restrepo Meta, se viene gestando desde hace 9 años. El primer paso fue "un fuerte trabajo de capacitación y sensibilización para toda la comunidad educativa, pues los temores no eran sólo de índole pedagógica, sino también con respecto a las condiciones propias de cada uno de los niños con NEE que ingresaban a la institución". Segundo, "aprender sobre el desarrollo cognitivo y afectivo de cada uno permite trazar una ruta de viaje y establecer las rutas pedagógicas y didácticas apropiadas para cada caso". Tercero, "diseñar estrategias como el manejo de clases individualizadas, utilización de sombras o acompañantes o la flexibilización curricular". (Camacho 2013).

Siguiendo con la autora, entre los resultados de este proceso se destaca que "incorporar nuevas estrategias pedagógicas al aula favorece a todos": a nivel profesoral, se forja un maestro más "creativo, optimista y paciente; cada uno tiene claro su papel y su importancia dentro del proceso de aprendizaje de los niños". En cuanto a la comunidad estudiantil, en 
general, "el compartir el día a día de clase, el recreo y las actividades propias de la institución ha marcado positivamente la vida de los niños regulares, quienes han aprendido a comprender y conocer mejor a los otros, a respetar las diferencias y a mejorar sus relaciones interpersonales".

(e) La inclusión en el Gimnasio Santa Rocío, en Bogotá, se basa en dos pasos: el primero consiste en caracterizar las barreras de aprendizaje; el segundo, en generar inclusión educativa según la caracterización realizada en el paso uno. Como se puede leer en http://www.gsantarocio.edu.co/gimnasio-primariabachillerato-bogota/Proyecto\%20Inclusion \%20-68 (s.f) "La inclusión brindada es para aquellos estudiantes que tienen: discapacidad cognitiva leve, trastorno de déficit de atención con hiperactividad leve -TDAH-, dificultades de lenguaje, falta de habilidades sociales, discapacidad motora, dificultades para el aprendizaje -proceso lectoescritor y pensamiento matemático-, dificultades de comportamiento, síndrome epiléptico de ausencias, déficit sensorio integrativo, fibrosis quística, discapacidad auditiva, síndrome de Asperger y retardo en el lenguaje.

Para cumplir con el objetivo de brindar una educación inclusiva, esta se realiza a través del compromiso de cada uno de los miembros de la comunidad educativa y se cuenta con un equipo interdisciplinario de apoyo interno y externo, compuesto por profesionales de la salud y profesionales psicopedagógicos para tener un acompañamiento e intervenciones más asertivas.

(f) En el proyecto de educación inclusiva del preescolar Carrizales, en Envigado - Antioquia, se realizó una etapa de sensibilización de las maestras frente al tema, una de construcción, donde se plantearon las necesidades de aula acuerdo a las características de cada grupo y a las necesidades e intereses de las maestras y por último una etapa de análisis de resultados (Serna y Flórez 2014).

(g) El objetivo principal del programa del MEN, Educación para todos como se puede leer en http://www.mineducacion.gov.co/1621/article-

141881.html (2007) es brindar inclusión educativa a través de la cobertura escolar, con programas que busquen la inclusión por medio de la eliminación de barreras que se encuentran en el sistema educativo e incluso en la misma sociedad, dando prioridad a la educación de poblaciones vulnerables.

(h) En la Institución Educativa San Sebastián, de La Plata, Huila, de acuerdo con Atehortúa y otros autores (2009), el Proyecto Aula de Apoyo se centra en educación inclusiva para estudiantes con discapacidad cognitiva -de retardo mental, síndrome de Down y parálisis cerebral- y limitación física, articulando este propósito con su direccionamiento estratégico -Misión y Visión-, en donde toman como pilar de soporte al padre de familia, "capacitándolo y sensibilizándolo sobre el compromiso adquirido con la institución educativa para la formación integral de su hijo". Entre las estrategias implementadas sobresalen: 1) Cooperación con entidades que garantizan recursos técnicos, terapéuticos $y$ financieros; 2) capacitación, asesoría y estrategias pedagógicas que permitan al maestro integrador interactuar en el aula y generar espacios de educación inclusiva y equitativa para con todos los estudiantes; 3) Asesoría y capacitación a padres y madres de familia de estudiantes con NEE en diversas estrategias de enseñanza de los mismos, para que sean un apoyo permanente al trabajo realizado en la institución y 4) Evaluación psicopedagógica y atención a los estudiantes con NEE.

(i) En la Institución Educativa Villa Santana, en Pereira, Risaralda, la inclusión se desarrolla en el marco de una investigación en la que se realiza una serie de observaciones de las "prácticas pedagógicas de los docentes en las clases de Ciencias Naturales, con el fin de identificar estrategias usadas por ellos para facilitar la inclusión de los escolares con discapacidad cognitiva. Esto permite recolectar información necesaria $y$ pertinente del quehacer en el aula de los docentes y estudiantes" (Rosero y Jaramillo 2012).

Los procesos pedagógicos llevados a cabo en el plantel educativo se caracterizan por ser, según las autoras referenciadas, "estrategias derivadas de enfoques constructivistas y otras de individuación de la enseñanza". Así mismo, cuenta con el aula de apoyo y la estrategia de la metodología GEEMPA y la sensibilización "a los docentes de la importancia de atender adecuadamente a los estudiantes con barreras en la participación y el aprendizaje". No obstante, existen carencias en cuanto a recursos y formación para atender a la diversidad estudiantil, no permitiendo que el entorno se adapte al estudiante sino el estudiante al medio.

La institución ha realizado una serie de estrategias pedagógicas en el área de las Ciencias Naturales, tales como: "adaptaciones curriculares, por medio de diversas herramientas como canastas educativas, juegos pedagógicos, vivencias, libros, entre otros, y el acompañamiento y asesoría del docente de apoyo" (Rosero y Jaramillo 2012).

\subsection{Instituciones de Educación Superior}

Son varias las universidades que han implementado diversas estrategias para la inclusión educativa, entre las que destacan flexibilización de currículos, 
orientación académica, nuevas tecnologías y mejor infraestructura. En el 2005 se creó la Red Colombiana de Universidades por la Discapacidad RCUD- cuyo fin principal es socializar estrategias de educación inclusiva. A continuación, algunas de esas experiencias:

(a) La Universidad Javeriana, en Santiago de Cali, (El Espectador 2015; Vicerrectoría del medio universitario de la Pontificia universidad Javeriana s.f.) determina el inicio a la inclusión dividido en tres etapas:

En la Etapa 1, entre 2009 y el 2011, se crea el Comité Institucional para implementar una serie de iniciativas que ya se venían gestando con relación a la inclusión de personas en condición de discapacidad física y sensorial. Se compran la impresora Braille y diez licencias del programa JAWS, se capacita al personal de la biblioteca, se realiza el primer censo de estudiantes y profesores con alguna discapacidad motora o sensorial para facilitar estrategias educativas y se crearon los fondos de becas para Grupos Étnicos y Fe y Alegría.

En la Etapa 2, entre 2012 y 2014, se inicia la ejecución del proyecto Fortalecimiento de estrategias formativas para la inclusión de personas con discapacidad, grupos étnicos y población con vulnerabilidad económica. Como resultado, se publican cinco documentos sobre la caracterización de factores que inciden en el acceso, retención y egreso de estudiantes con discapacidades, etnia diversa y vulnerabilidad socioeconómica; se crearon dos cátedras de formación docente; se realizaron Jornadas de sensibilización con la Comunidad Educativa; se incrementó el material bibliográfico en la biblioteca; se definieron lineamientos para una política institucional y la Universidad se vinculó a las redes universitarias locales y nacionales. Adicional a ello, se valoró la planta física de la Universidad con base en la norma técnica colombiana NTC4140.

En la Etapa 3, en 2015, se implementó la política del gobierno nacional con el programa "Ser Pilo Paga" y, a nivel interno se hizo la renovación del Comité Institucional de Educación Inclusiva. Así mismo, se participó activamente en tres eventos de ciudad: Encuentros itinerantes; 3er Coloquio Colombiano de Investigación en Discapacidad y Asamblea Nacional de Universidades por la Discapacidad.

Todo lo anterior conllevó a concebir la inclusión a nivel institucional. Primero, se procedió a la implementación de mesas interdisciplinarias de trabajo para analizar políticas de integración institucional. Segundo, se identificaron las personas en situación de discapacidad, se realizaron foros con el fin de compartir experiencias educativas y políticas de inclusión. Por último, hubo capacitación a la planta profesoral para fortalecer sus metodologías.

(b) La Universidad del Rosario (El Espectador 2015) ha implementado un acompañamiento desde el proceso de admisión hasta que el estudiante culmine el programa académico. También ha adquirido softwares especializados y dispositivos que codifican el sistema braille y facilitan el desarrollo de trabajos y exámenes para aquellos estudiantes en condición de discapacidad visual.

(c) La Universidad del Bosque (El Espectador 2015) adaptó los procesos académicos e implementó un acompañamiento individual. Con relación a los espacios físicos, los edificios y las aulas de clase se ajustaron a las normas internacionales, permitiendo así, que estudiantes con diferentes tipos de discapacidad física, sensorial y motora se gradúen de carreras como ingeniería, música, psicología y pedagogía infantil.

(d) La Universidad Nacional (El Espectador 2015) se reconoce por sus programas para la inclusión de poblaciones vulnerables por medio de beneficios en los costos de matrículas, además de, según el rector Ignacio Mantilla, implementar cambios en la docencia, la investigación, la administración y la movilidad. Los exámenes de admisión están diseñados también para personas en condición de discapacidad.

(e) La Universidad Escuela Colombiana de Carreras Industriales -ECCl- cuenta con prácticas educativas de inclusión orientadas al acceso a la educación de personas con discapacidad auditiva "generando estrategias desde el ámbito académico y pedagógico que implica el diseño de currículos, metodologías didácticas, material didáctico y sistemas de evaluación, adecuación de los espacios físicos y de la estructura organizacional, personal administrativo, de servicio y cuerpo docente" (Universidad Escuela Colombiana de Carreras Industriales -ECCl s.f.).

Con el fin de identificar las prácticas que pudiesen ser las más apropiadas a la hora de iniciar un proyecto de accesibilidad en una institución educativa se han caracterizado y agrupado las estrategias implementadas por cada uno de los casos expuestos, los cuales se detallan en la Tabla 1.

\section{HERRAMIENTAS DE SOFTWARE QUE APOYAN ESPACIOS INCLUSIVOS}

En relación a herramientas que apoyen espacios inclusivos, la investigación se centra en tres aspectos: la accesibilidad, la creación de recursos educativos accesibles y el uso de metodologías de diseño para todos. En este sentido, entre las 
principales herramientas están: la Web Content Accesibility Guidelines, la Web Content Authorin Tool y el Universal Design for Learning -UDL.

La Web Content Accesibility Guidelines -WCAG, por sus siglas en inglés- brinda pautas para el diseño de sitios web orientados a la accesibilidad de todos los usuarios, de tal manera que el contenido "sea accesible para un mayor número de personas con discapacidad, incluyendo ceguera y baja visión, sordera y deficiencias auditivas, deficiencias del aprendizaje, limitaciones cognitivas, limitaciones de movilidad, deficiencias de habla, fotosensitividad y combinaciones de las anteriores e incluso también ayudan a que el contenido sea más usable para las personas mayores" (Cadwell et al. 2009). De igual manera, estos estándares proporcionan facilidad y agilidad en la búsqueda de información en la Web; entiéndase información como texto, imágenes, videos, sonidos, estructuras de programación y presentación, entre otras. Tal como lo expresa ([Lawton 2017), los principios de accesibilidad provistos por la WCAG están encauzadas a simplicidad y comodidad en cuanto a los criterios de perceptibilidad, operatividad, entendible y robusto.

Tabla 1. Estrategias implementadas

\begin{tabular}{|c|c|}
\hline Estrategia & Descripción \\
\hline \multirow{7}{*}{$\begin{array}{l}\text { Sensibilización } \\
\text { y capacitación }\end{array}$} & $\begin{array}{l}\text { Equipos de apoyo que pueden ser conformados por profesionales de la salud, profesionales } \\
\text { pedagógicos, profesores, estudiantes y padres de familia }\end{array}$ \\
\hline & Sensibilización con el equipo de apoyo, maestros, padres de familia y compañeros de clase \\
\hline & $\begin{array}{l}\text { Capacitación de profesores sobre las condiciones y caracteristicas de cada caso, } \\
\text { metodologías pedagógicas y didácticas }\end{array}$ \\
\hline & Capacitación de personal administrativo \\
\hline & Capacitación de personal de servicios \\
\hline & Capacitación a padres de familia en estrategias y enseñanzas pedagógicas \\
\hline & Acompañante del proceso cognitivo (compañero de estudio $y / 0$ profesor) \\
\hline \multirow{3}{*}{ Curricular } & Flexibilización curricular (cartillas especiales) \\
\hline & No hay diferencias curriculares \\
\hline & $\begin{array}{l}\text { Implementación de metodologia GEEMPA (se cimienta en la lúdica como estrategia para } \\
\text { cuativar la atención del estudiante) }\end{array}$ \\
\hline \multirow{2}{*}{$\begin{array}{l}\text { Softwares y } \\
\text { dispositivos }\end{array}$} & Adquisición de dispositivos de lectura y escritura Braille \\
\hline & Compra de software lector de pantalla para personas con problemas de visión \\
\hline \multirow{2}{*}{ Económico } & Creación de fondos y becas \\
\hline & Cooperación con entidades que garantizan recursos técnicos, terapéuticos y financieros \\
\hline \multirow{6}{*}{ Institucional } & Creación de cátedras concernidas con el tema \\
\hline & Definición de lineamientos institucionales a través de mesas de trabajo interdisciplinarias \\
\hline & Vinculación con redes locales y nacionales \\
\hline & Adecuación de la planta física \\
\hline & Participación en eventos relacionados con la educación inclusiva \\
\hline & Creación de la Red Colombiana de Universidades por la Discapacidad (RCUD) \\
\hline
\end{tabular}

De otro lado, de acuerdo con Corredor (2015), el Web Content Authoring Tool es un software para la creación de contenidos, usualmente en multimedia para la World Wide Web. Algunos ejemplos de herramientas de creación de contenido son Shareable Content Object Reference Model SCORM, por sus siglas en inglés-, HTML5, entre otros.

SCORM es un conjunto de estándares que permite crear contenido de pedagógico de manera estructurada. Esta robustez facilita su uso e implementación por centros educativos e incluso organizaciones. No obstante, con el transcurso del tiempo y la evolución tecnológica, se concibe TIN CAN API, conocido ahora como Experience API xAPI-como "un nuevo estándar para las aplicaciones de e-learning que estructura y permite almacenar las interacciones de una persona ante distintas actividades de aprendizaje online. A través de este programa, sistemas muy diferentes pueden comunicarse entre sí en forma segura e intercambiar los flujos de actividades de una persona o un grupo de personas mientras interactúan con diversas tecnologías y contenidos" (http://tincanapi.com/ s.f.). 
Las herramientas de creación de e-learning HTML5 permiten el soporte en dispositivos móviles, tales como smarthphones y tablets, permitiendo que los usuarios lleven sus cursos de e-learning consigo en estos dispositivos, en vez de requerir computadores para su uso. Incluso, "los cursos de elearning en HTML5 usan menos espacio en las CPU y menor consumo de batería, lo cual significa que son más eficientes" (Pappas 2017). Algunos de los programas más utilizados en la industria del elearning son: Adobe Captivate 9, Elucidat, Articulate Storyline 360, Gomo Learning, ITyStudio, Obsidian Black, BranchTrack, Easygenerator, Adapt Authoring Tool, y Adobe Animate CC.

La Metodología Universal Design for Learning -
UDL, por sus siglas en inglés- consiste en "una serie de principios para el desarrollo de currículos que den a todos los individuos igualdad de oportunidades para aprender" (National Center Universal Design for Learning -UDL 2017). Los retos del mundo pedagógico de la actualidad consisten en crear flexibilidad en el proceso de aprendizaje, dada la heterogeneidad de los estudiantes. No obstante, estos principios aplican para estudiantes de educación presencial, semi presencial y virtual, de tal manera que el currículo a través de las metas, métodos, materiales y entregables sean personalizados y así, ser aplicados y entendidos por todos.

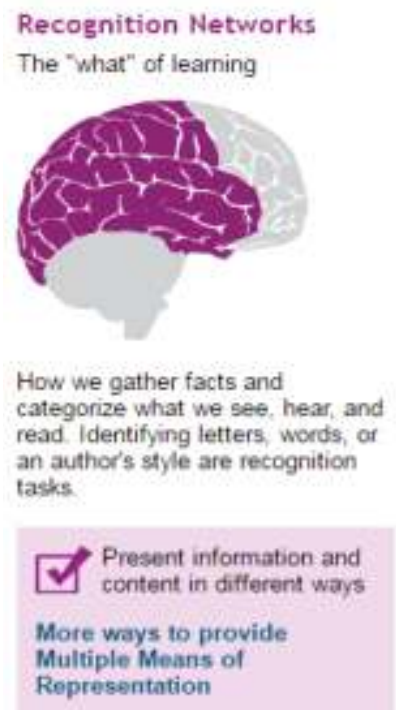

Figura 1. Los tres principios del UDL

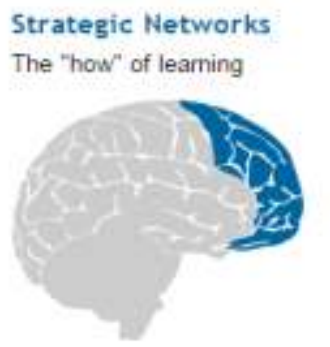

Planning and performing tasks. How we organize and express our ideas. Writing an essay or solving a math problem are strategic. tasks

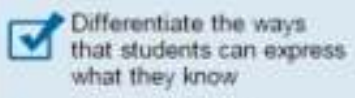

More ways to provide Multiple Means of Action and Expression

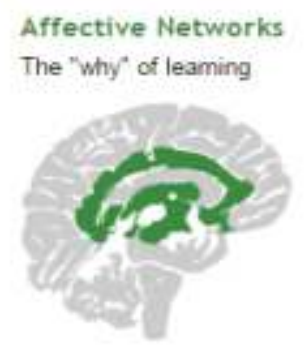

How learners get engaged and stay motivated. How they are challenged, excited, or interested. These are affective dimensions.

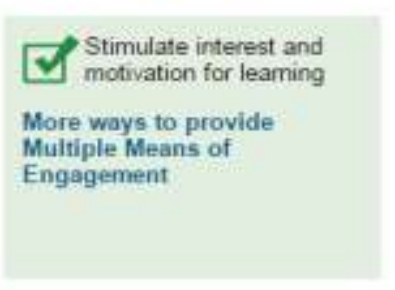

\section{Fuente. CAST}

UDL se basa en tres principios (2017), los cuales se muestran en la Figura 1 , y se explican a continuación.

EI Principio I consiste en proporcionar múltiples medios de representación -el 'qué' del aprendizajepuesto que los estudiantes aprenden de distintas maneras, de acuerdo con los diferentes tipos de discapacidades. Por ejemplo: personas con discapacidades sensoriales; discapacidades de aprendizaje; lenguaje 0 diferencias culturales, requieren mecanismos diferentes. Resulta imperativo tener en cuenta los estilos de aprendizaje: visual, auditivo y kinestésico.

EI Principio II consiste en proporcionar múltiples medios de acción y expresión -el 'cómo' del aprendizaje- debido a que los estudiantes difieren uno de otro en la forma en la que pueden expresar sus conocimientos $y$, por ende, ser evaluados. Por tal razón, es necesario ofrecer variedad de medios en los cuales los estudiantes pongan en práctica sus saberes.

El Principio III se basa en proporcionar múltiples medios de compromiso -el 'por qué' del aprendizaje- La motivación cumple un papel sumamente importante en el proceso de aprendizaje y los estudiantes difieren unos a otros de la manera en que se comprometen y motivan; cada uno con justificaciones diferentes tales como la neurología, la cultura, vivencias, la relevancia personal, la subjetividad y el conocimiento de fondo. 


\section{ConClusiones}

El diseño tiene un carácter de universalidad, de inclusión y al igual que una edificación física, que permita el libre ingreso, libre circulación y flujo normal del personal. El ambiente de aprendizaje debe ser adaptable y flexible a las necesidades y diferencias de las personas, resultando en la eliminación de las barreras que obstaculizan el proceso de enseñanza-aprendizaje.

Existen cambios tecnológicos que favorecen los nuevos paradigmas de educación, donde la diversidad estudiantil es la fuente que potencializa la inclusión. El objetivo de la educación del siglo XXI es que los estudiantes se conviertan en maestros expertos del aprendizaje a través de un autoconocimiento de sí mismos, soportado por leyes gubernamentales que promuevan no sólo la educación inclusiva sino también la inclusión social de aquellas personas que presentan alguna discapacidad.

Con relación al trabajo futuro, se realizará una profundización de la metodología UDL aplicada a casos en Colombia, con el fin de analizar las estrategias de inclusión incorporando esta metodología.

\section{REFERENCIAS}

Atehortúa, A., García, F., \& Muñoz, M. C. (2009). Proyecto de Inclusión Educativa de la Insitución Educativa San Sebastián. La Plata, Huila. Recuperado de https://es.scribd.com/doc/74227604/PROYECTOINCLUSION-EDUCATIVA

Calwell, B., Cooper, M., Guarino, L., \& Vanderheiden, G. (2008). Web Content Accessibility Guidelines (WCAG) 2.0. Recuperado http://www.sidar.org/traducciones/wcag20/es/

Camacho, C. (24 Mar 2013). El Espectador. Recuperado de http://www.elespectador.com/noticias/educacion/colegios -incluyentes-articulo-412347

Corredor, C. M. (2013). Framework for detection, assessment and assistance of university students with dyslexia and/or reading difficulties (Doctoral dissertation, Universitat de Girona).
El Espectador. (28 de marzo de 2015). Espacios para la inclusión. Bogotá. Recuperado

de https://www.elespectador.com/noticias/educacion/espaci os-inclusion-articulo-552057

Gimnasio Santa Rocio. (s.f.). Gimnasio Santa Rocio. Recuperado de http://www.gsantarocio.edu.co/gimnasio-primariabachillerato-bogota/Proyecto\%20Inclusion\%20-68

Lawton Henry S. (2017). Web Content Accessibility Guidelines (WCAG) Overview. Recuperado de https://www.w3.org/WAl/intro/wcag

Ministerio de Educación Nacional - MEN. (2007). Educación para todos. Recuperado de http://www.mineducacion.gov.co/1621/article141881.html

Ministerior de Educación Nacional - MEN. (2009). Educación inclusiva con calidad. Bogotá. Discapacidad Colombia. (s.f.). Discapacidad Colombia. Recuperado de http://discapacidadcolombia.com/index.php/articulosdiscapacidad/educacion-inclusiva/2-uncategorised/211bienvenidos-a-discapacidad-colombia-2

National Center On Universal Desing for Learning -UDL. (2017). UDL Implementation: A Tale of Four Districts. Recuperado de http://www.udlcenter.org/implementation/fourdistricts

Pappas, C. (2017). elearning Industry. Recuperado de https://elearningindustry.com/the-ultimate-list-of-html5elearning-authoring-tools

Rosero, D. M., \& Jaramillo, A. M. (2012). Proceso de inclusión educativa de escolares con discapacidad cognitiva en el área de las ciencias naturales de los grados $1^{\circ}, 2^{\circ}$ y $3^{\circ}$ de primaria. Pereira: Universidad Tecnológica de Pereira. Recuperado http://recursosbiblioteca.utp.edu.co/tesisd/textoyanexos/3 7192886132R816.pdf

Serna Cadavid, L. M., \& Flórez Restrepo, L. (2014). Intervención pedagógica, para la inclusión escolar de niños que presentan Necesidades Educativas Especiales. Preescolar Carrizales (Doctoral dissertation, Corporación universitaria Lasallista).

TinCanAPI. (s.f.) TincanAPI. Recuperado de http://tincanapi.com/.

UNESCO. (1994). Declaración de Salamanca y su marco de acción para las necesidades educativas especiales. Salamanca.

Universidad Escuela Colombiana de Carreras Industriales - ECCI. (s.f.). Programa de inclusión. Recuperado de https://www.ecci.edu.co/es/Bogota/programa-deinclusion-191?language content entity=es

Vicerrectoría del Medio Universitario de la pontificia Universidad Javeriana de Cali. (s.f). Proyecto transversal educación inclusiva. Recuperado de https://www.javerianacali.edu.co/sites/ujc/files/node/fielddocuments/field_document_file/proyecto_educacion_incl usiva.pdf 